\title{
Las percepciones estudiantiles de la escuela secundaria en Latinoamérica. Vínculos, pertenencia y valoración del conocimiento escolar en la modernidad avanzada
}

\author{
Student Perceptions of Secondary School in Latin \\ America.Relations, Belonging, and Valuation of \\ School Knowledge in Late Modernity
}

\section{Daniel Pedro Míguez}

Universidad Nacional del Centro de la Provincia de Buenos Aires, Argentina.

\section{Resumen}

\begin{abstract}
Este artículo examina las percepciones de la escuela que tienen estudiantes de nivel secundario en América Latina. Se comparan las percepciones entre distintos países de la región y también entre diferentes estratos sociales. A partir de estas comparaciones, se ponen a prueba las tesis que han guiado más frecuentemente este campo de investigación. Estas proponen que, producto de los cambios culturales y tecnológicos introducidos por la modernidad tardía, existiría una desvalorización de la escuela secundaria, que se profundizaría en los estratos más pobres. Utilizando datos de las pruebas PISA 2012, se pone en evidencia que la mayoría de los estudiantes latinoamericanos mantiene una visión positiva de la escuela, aunque con algunos matices. Estos sugieren que la experiencia escolar de los estudiantes secundarios es compleja, y presenta algunas ambigüedades y ambivalencias, pero no confirman la preeminencia de visiones negativas de la educación media.
\end{abstract}

Palabras clave: educación; percepciones; estudiantes; Latinoamérica; modernidad.

\section{Correspondencia a:}

Daniel Pedro Míguez

Instituto de Geografía, Historia y Ciencias Sociales. Consejo Nacional de Investigaciones Científicas y Técnicas y Universidad Nacional del Centro de la Provincia de Buenos Aires, Argentina.

Lanza del Vasto 658 / (7000) Tandil. Provincia de Buenos Aires, Argentina. dpmiguez@fch.unicen.edu.ar

Esta investigación fue posible gracias a un subsidio de duración plurianual del Consejo Nacional de Investigaciones Científicas y Técnicas de Argentina.

(c) 2021 PEL, http://www.pensamientoeducativo.org - http://www.pel.cl 


\section{Summary}

This paper examines the perceptions that Latin American secondary-level students have of their schools. These perceptions are compared across various countries in the region and between different social strata. Based on these comparisons, the paper tests the theses that have most frequently guided research in this field. The core of these theses is that, as a result of the cultural and technological changes introduced by late modernity, there is a devaluation of secondary education among high school students, which deepens among the poorest social strata. Based on data from the 2012 PISA tests, the study demonstrates that the majority of Latin American students have a positive view of the school, although with some nuances. These suggest that the school experience of secondary students is complex and presents certain ambiguities and ambivalences. However, these do not imply the preeminence of negative views of secondary education among Latin American students.

Keywords: education; perceptions; students; Latin America; modernity.

Este artículo examina, desde una perspectiva comparativa, las percepciones de la escuela secundaria que tienen los estudiantes de nivel medio en América Latina. Con base en los datos de las pruebas PISA del año 2012, se comparan las percepciones de estudiantes de distintos países y también de diferentes estratos sociales. En función de estas comparaciones, se analizan algunas de las premisas que han guiado la investigación sobre la forma en que los cambios introducidos por las fases más avanzadas de la modernidad habrían afectado la percepción de la escuela que tienen los estudiantes de nivel medio o secundario en Latinoamérica.

En términos generales, los estudios sobre los efectos de la modernidad avanzada en la experiencia escolar suponen un vínculo crecientemente conflictivo entre los estudiantes y la escuela (Perelman, 1992; Duschatzcky y Corea, 2002; Dubet y Martuccelli, 1997; Tenti, 2014). Este se habría originado en dos procesos relacionados. Uno de ellos sería más propio (aunque no exclusivo) de sectores con mayores niveles de integración social y con un mayor acceso al conocimiento y a los cambios tecnológicos introducidos en esta fase de la modernidad. Otro de esos procesos afectaría a sectores sociales menos integrados, y con más dificultades para acceder a esos saberes y tecnología.

En ambos casos, los estudiantes experimentarían un extrañamiento respecto de la institución escolar. Sin embargo, en el primero, este derivaría de la influencia de los avances tecnológicos y cognoscitivos sobre las percepciones de ese sector social respecto de los saberes y las modalidades de vinculación y enseñanza tradicionalmente propuestas por la escuela. En el segundo, en cambio, serían los crecientes niveles de exclusión a los que quedarían sometidos algunos sectores de la sociedad los que incrementarían la distancia respecto de ella.

Si bien estas premisas conformaron una buena parte de las investigaciones sobre las percepciones de la escuela en la modernidad avanzada, estas no siempre fueron confirmadas por los estudios que analizaron más empíricamente sus efectos en América Latina. Como veremos, algunos de estos estudios confirman que los estudiantes latinoamericanos perciben sus relaciones con los docentes como conflictivas, y a los saberes y formas de pertenencia propuestos por la escuela, como irrelevantes. Sin embargo, otros encuentran grupos estudiantiles en los que predomina una valorización positiva de la escuela y de los vínculos con las autoridades educativas. Dado que la mayor parte de estos resultados surgen de estudios de caso, es difícil establecer si las diferencias encontradas representan tendencias generales o se originan en las particularidades de los grupos estudiados. 
En este artículo, intentaremos realizar un aporte a este campo de investigación desarrollando una perspectiva comparativa entre países y estratos sociales mediante una aproximación estadística. Si bien las limitaciones que presentan estos datos no permiten determinar totalmente el origen de las diferencias que surgen de los estudios de caso, sí sugieren algunas tendencias generales y formas de refinar las premisas que han guiado estas investigaciones para mejorar nuestra comprensión de esos matices.

Tomando en cuenta estos objetivos, en la próxima sección presentaremos en más detalle los avances alcanzados por la investigación sobre las percepciones estudiantiles de la escuela secundaria en América Latina. Posteriormente, explicitaremos las características de las fuentes de datos que utilizamos en nuestro estudio, y los métodos y variables que empleamos en él. Luego, expondremos los resultados que surgen de su análisis. Finalmente, discutiremos los matices que estos sugieren respecto de las premisas que orientaron las investigaciones sobre el tema.

\section{La experiencia escolar en la modernidad avanzada}

Quienes han caracterizado las fases más recientes de la modernidad la describen a partir de la asociación entre nuevas formas de sociabilidad y pertenencia social, y el desarrollo de nuevos conocimientos y nuevas tecnologías de la producción y de la comunicación. Puesto brevemente, los cambios tecnológicos y cognoscitivos estarían asociados a un debilitamiento de las regulaciones institucionales de las relaciones interpersonales (familiares, de amistad, laborales, etc.) y, también, a una transformación de las formas de organización del proceso productivo y a una retracción de los dispositivos de seguridad social, generando mayores grados de incertidumbre personal e inestabilidad laboral y económica (García Canclini, 1995; Castel, 1997; Giddens, 1997; Sennet, 1998; Lewkowicz, 2004)․․

Tomando en cuenta el efecto dispar de estas transformaciones en diversos sectores sociales, la bibliografía que indaga sobre la experiencia escolar en esta etapa ha dado cuenta de dos procesos asociados: uno que habría afectado más a los sectores socialmente integrados y otro, a aquellos con mayor nivel de exclusión.

En el primer caso, los estudios señalan que los cambios propios de la modernidad avanzada habrían influido en el sistema educativo al menos de tres maneras: transformando los fundamentos de las modalidades tradicionales de autoridad y orden escolar; generando intereses y formas de pertenencia y sociabilidad juvenil distantes de los que caracterizan a la escuela, e introduciendo una rápida obsolescencia del conocimiento y formas de instrucción escolar (Dubet y Martuccelli, 1997; Tenti, 2000, 2014; Bauman; 2005; Giacobbe y Merino, 2015).

Respecto de la primera cuestión, el carácter reflexivo que asumirían los vínculos interpersonales en la fase más avanzada de la modernidad habría transformado los fundamentos de la autoridad docente ${ }^{2}$. A diferencia de lo que sucedía en la escuela tradicional, en donde esta se legitimaba en el orden institucional, en el nuevo orden escolar la capacidad de liderazgo estaría fundada en la habilidad del docente para generar consenso entre sus estudiantes (Dubet, 2002, 2011; Martuccelli, 2009; 2017; Tenti, 2014).

La celeridad con que habrían ocurrido estas transformaciones y su carácter relativamente tácito o implícito no habría permitido que los docentes se formaran en esta nueva modalidad de ejercicio profesional. Así, el malestar se habría convertido en una suerte de rasgo característico de la docencia, y los vínculos entre docentes y estudiantes se habrían vuelto más indeterminados y conflictivos (Sánchez, 2006; Dubet, 2009; Giacobbe y Merino, 2015).

1. Por razones de espacio, resulta imposible presentar una caracterización exhaustiva de la modernidad avanzada aquí. Estos autores representan algunas de las teorías más relevantes y conocidas, sobre las que daremos algunas precisiones más adelante.

2. En lugar de estar regulados por pautas institucionales rígidas, en la modernidad avanzada los vínculos interpersonales serían objeto de un proceso reflexivo: evaluados y reelaborados en función del sentido que estos adquieren y del grado de satisfacción que proveen a las partes involucradas (Giddens, 1997). 
La segunda fuente de conflicto surgiría de la creciente incompatibilidad entre las formas de comunicación y pertenencia promovidas por la escuela y aquellas propias de los sectores juveniles (Martuccelli, 2009, 2017). Si bien parte de estas tensiones ya se había manifestado en las décadas intermedias del siglo XX (Coleman, 1961; Manzano, 2010), una nueva fase se produciría a partir de las postrimerías de ese siglo.

Como lo ha señalado Martuccelli (2009), en esta etapa las nuevas formas de comunicación en red promoverían modelos relacionales horizontales, donde no existen emisores y receptores predefinidos y jerarquizados. Asimismo, la cultura visual y oral que es propia de las nuevas tecnologías comunicacionales no proveería bases compatibles con la centralidad que la lectura y la escritura tienen en la práctica escolar. Finalmente, en la cultura juvenil predominaría la espontaneidad, la búsqueda del placer, la creatividad y la expresión como un fin en sí mismo. En cambio, la cultura escolar tradicional se caracterizaría por promover la abnegación, el cumplimiento del deber, la disciplina y el patriotismo como valores centrales. Así, las formas relacionales y comunicacionales características del dispositivo escolar no encontrarían un sustrato propicio entre las pautas de sociabilidad y pertenencia desarrolladas por los jóvenes.

Una tercera dimensión del conflicto resultaría de la rápida obsolescencia de las modalidades de enseñanza y saberes difundidos por la escuela (Perelman, 1992; Burbules y Torres, 2013). Esta caducidad no sería el resultado, tan solo, de que la información o conocimientos difundidos por la escuela resultarían superados por otros más avanzados, sino que el acceso inmediato y multisituado a la información mediante los nuevos dispositivos tecnológicos vaciaría de sentido la práctica tradicional de retenerla mediante la instrucción memorística centrada en las aulas (Martuccelli, 2016).

Como ya adelantamos, junto con estos cambios, las transformaciones tecnológicas y cognoscitivas introducidas por esta fase de la modernidad habrían profundizado los niveles de exclusión social de los sectores tradicionalmente relegados. Esto se debería, por un lado, a los crecientes niveles de calificación y acreditación necesarios para lograr una integración social plena. Aunque esta mayor exigencia habría sido acompañada por un incremento de la escolarización, incluso de los estratos tradicionalmente más relegados (aunque persisten importantes diferencias entre países al respecto), esto no necesariamente implicaría mayor igualación.

El incremento del acceso de la población a niveles cada vez más altos de escolarización, si bien, en algunos sentidos, habría permitido mayor cercanía entre la escuela y las pautas de sociabilidad de los sectores populares, no resolvió plenamente el problema. Similar al suplicio de Tántalo, quien veía alejarse su objetivo cada vez que estaba por alcanzarlo, esta suerte de masificación del acceso a la escuela incrementaría los niveles educativos necesarios para lograr la plena integración social (Susmel, 2012). Así, a medida que más sectores accedían a niveles más altos del sistema educativo, estos perdían su valor relativo. Asociado a eso, las transformaciones que debía sufrir la institucionalidad escolar para incorporar a sectores tradicionalmente excluidos harían más débil su capacidad de transmitir las calificaciones necesarias para ello (Tenti, 2000).

En este contexto, surgiría una creciente diferencia entre establecimientos que mantendrían o incrementarían su capacidad de transmitir esas calificaciones y aquellos que las verían degradarse. El acceso diferencial a estas instituciones por sector social mantendría o incluso profundizaría los niveles de desigualdad social, pese al acceso incremental a la educación de los sectores tradicionalmente relegados (Dubet, 2009). En este sentido, el acceso a la escuela y la mayor familiaridad con los roles desempeñados en ella, no garantizaría per se el acceso a las competencias exigidas por el mercado laboral (Dubet, 1995). De este modo, pese a su masificación, el sistema educativo habría mantenido importantes niveles de segregación entre estratos sociales, reproduciendo las asimetrías de origen entre los estudiantes (Merle, 2012).

Otra de las características de la modernidad avanzada que habría profundizado las desigualdades es la incremental retracción de los tradicionales dispositivos de seguridad social (el sistema previsional, la estabilidad laboral, la salud pública, etc.). Esta retracción habría dado al mercado de consumo y trabajo un lugar prioritario como 
locus de la integración social (García Canclini, 1995; Lewkowicz, 2004). Así, los sectores sin un acceso fluido a los mismos, incluidos los jóvenes de los estratos más pobres, enfrentarían un proceso "descivilizatorio" (Tenti, 2014, p. 7) o de "desubjetivación" (Duschatzky y Corea, 2002, p. 23).

Es decir, encontrarían dificultades crecientes para elaborar identidades socialmente reconocidas que faciliten su integración social, incluso mediante los dispositivos propios de las fases clásicas de la modernidad: la familia, el trabajo estable, y, claro está, la escuela. La multiplicación del conflicto con el orden normativo de la escuela, así como el surgimiento de nuevas formas de agresión entre estudiantes o de estos hacia los docentes, constituirían algunos de los emergentes de este tipo de transformación (Duschatzcky y Corea, 2002; Abramovay, 2003; Dubet, 2009).

En síntesis, este conjunto de estudios también planteó que las condiciones introducidas por la modernidad avanzada propiciaban vínculos crecientemente conflictivos entre los jóvenes y la escuela. No obstante, desde esta perspectiva, no se trataba de que los nuevos conocimientos y tecnologías alejasen a los jóvenes de ella, sino que, para este sector social, la imposibilidad de acceder a ellos incrementaba sus niveles de exclusión, al punto que la experiencia escolar misma se volvía inalcanzable o ajena.

\section{Datos en tensión}

Ahora bien, si la premisa de que, por distintas vías, la modernidad avanzada promovería vínculos conflictivos entre los estudiantes y la escuela influyó en una buena parte de la investigación en el campo, esta no fue siempre confirmada por los datos relevados por diversos estudios en Latinoamérica. No es que estos presentasen siempre evidencia incompatible con esta premisa general, pero si bien algunos datos resultaron coincidentes con ella, otros revelan una complejidad que obliga a repensarla más detenidamente.

Por ejemplo, un texto de Miranda López (2012) revela que estudiantes de Argentina, Chile, México y Uruguay experimentan un significativo malestar respecto de las normas de conducta que propone la escuela. Asimismo, los estudiantes de estos países perciben a la escuela como exigente, alejada de sus intereses, y a la relación con los docentes como distante y fría (Miranda López, 2012). Así, desde una perspectiva comparativa, el texto encuentra evidencia compatible con la premisa que plantea un extrańamiento entre las formas de conocimiento, vinculación y pertenencia que propone la escuela y las que desarrollan los y las estudiantes.

Los datos presentados en este trabajo son, a su vez, coincidentes con los de otras investigaciones hechas sobre ciertos países. Para el caso de Chile, por ejemplo, Sapiains y Zuleta (2001) o Metiffogo y Sepúlveda (2005) presentan entrevistas en las que los estudiantes también manifiestan malestar en su relación con los docentes. Por su parte, Tapia, Pantoja y Fierro (2010) -en el caso de México-, Dussell, Brito, Nuñez y Litichever (2006) y Saintout (2007) - en el caso argentino- o Rodríguez (2002), Calicchio, de León, Gutiérrez, Lorenzo y Radakovich (2004), y Richards (2009) -en el caso de Uruguay- muestran que los estudiantes de esos países también manifiestan un extrañamiento o distanciamiento respecto de la escuela.

Sin embargo, en paralelo, otros estudios revelan matices. En el caso de Colombia, De Giraldo y Mera (2000) encuentran que entre los estudiantes de ese país la escuela genera sensaciones de satisfacción en la medida en que logren establecer buenos vínculos con sus compañeros y avanzar en los estudios. A su vez, el análisis de Meza (2017) sobre la realidad mexicana muestra percepciones variadas. Mientras en algunos casos los estudiantes rechazan la rigidez del sistema disciplinario, incluso esos mismos estudiantes valoran otros aspectos del orden institucional. Por ejemplo, sienten que cierta regulación de la apariencia y de las formas de vinculación que tienen lugar en la escuela les permiten adquirir una identidad socialmente valorada. 
En el caso de Argentina, varios trabajos sugieren la existencia de percepciones complejas o ambivalentes entre los estudiantes, aunque no necesariamente contradictorias. Específicamente, muestran que distintos estudiantes pueden experimentar la escuela de maneras diversas o valorar la misma experiencia de distintas formas. Por ejemplo, un estudio realizado por la Fundación SES (2018) con base en una encuesta de 900 casos mostró que, en general, los y las estudiantes que participaron en el estudio percibían a la escuela como un lugar agradable. Solo una minoría vinculaba la escuela a experiencias negativas, como la violencia, el aburrimiento o la sobre exigencia. A su vez, otra investigación sobre Argentina encuentra estudiantes que valoran aspectos de la experiencia escolar que aparecían como fuente de conflicto en varios de los anteriores, como "el vínculo con los profesores que les enseñan y acompañan; y [...] la exigencia, tanto en lo que hace al nivel de estudios como a las normas en general" (Llinás, 2009a, p.96). Notoriamente, entre estos estudiantes la crítica a la escuela no muestra un distanciamiento con el modelo institucional tradicional, sino el reclamo de que esta se ajuste a él. Los estudiantes cuestionan los problemas de infraestructura que presentan algunas escuelas y los déficits de aquellas que tienen un bajo nivel académico y que generan desigualdad respecto de otras de mayor nivel (Llinás, 2009b).

Interesantemente, la comparación de percepciones de estudiantes argentinos de distintos estratos sociales revela cierta homogeneidad en la percepción de la escuela, aunque con algunos matices. En todos los estratos la escuela aparece como un lugar de pertenencia y transmisión de hábitos y valores relevantes. Sin embargo, entre los sectores de menores recursos es vista como protección de los contextos y hábitos que inducen a la marginalidad, como el consumo de drogas o el ingreso a bandas delictivas. Por su parte, en las de mayores recursos es percibida como la transmisora de las predisposiciones cognitivas y actitudinales que reproducirán la pertenencia social de las nuevas generaciones (Tiramonti y Minteguiaga, 2007).

En síntesis, el campo de estudios sobre las percepciones estudiantiles de la escuela media o secundaria presenta una interesante tensión. Mientras que algunos trabajos encuentran datos que confirman la premisa de un proceso de extrańamiento entre los estudiantes y la escuela, otros revelan percepciones que siguen colocando a la educación secundaria en un lugar relevante como espacio de sociabilidad, pertenencia y de acceso al conocimiento. Como hemos señalado, si bien los datos estadísticos que presentamos a continuación no permiten saldar plenamente esta cuestión, sí otorgan una visión más generalizada sobre la percepción de la escuela que tienen estudiantes de nivel medio o secundario en América Latina.

\section{Fuentes, variables y métodos}

Los datos que utilizamos en este estudio provienen del cuestionario a estudiantes implementado en las pruebas PISA 2012. Elegimos este año porque, debido a fallas en el marco muestral, los datos de 2015 no eran representativos para el caso de Argentina (OECD, 2016), y los del 2018 aún no se habían publicado cuando realizamos este estudio.

Es importante aclarar que las bases PISA no contienen datos para todos los países de América Latina. Solo incluyen a Argentina, Brasil, Chile, Colombia, México, Perú y Uruguay. Por otro lado, las pruebas incluyen a estudiantes de 15 años que se encuentren dentro del sistema educativo. Así, es representativa de un grupo específico de estudiantes, que no solo se caracteriza por su composición etaria. Dado que en muchos países la deserción escolar se produce mayormente en los sectores de menores ingresos y antes de esa edad, las pruebas seguramente sub representan a los estratos sociales más pobres (Krüger, 2019).

Pese a estas limitaciones, la base utilizada contiene variables que permiten asir algunas dimensiones de la experiencia escolar que han sido consideradas como afectadas por las fases más avanzadas de la modernidad: los vínculos docente-alumno/a, los sentimientos de pertenencia a la escuela y la valoración de los saberes escolarizados. 
Sin embargo, es importante reconocer que solo lo hacen de manera parcial. Al realizar preguntas cerradas, las encuestas tienden a amoldar las opiniones de los entrevistados a un conjunto limitado de respuestas que, en cierta medida, limitan los matices o ambigüedades que captan (Cicourel, 1964).

Por este motivo, las aproximaciones cuantitativas a los fenómenos intersubjetivos tienden a registrar sus dimensiones emergentes, pero son menos sensibles a aquellas que se encuentran subyacentes a ellas. Es decir, reconocen lo que se manifiesta a nivel de la conciencia discursiva, pero que no representa totalmente las dimensiones preconscientes que de todas formas constituyen esas percepciones ${ }^{3}$. Como mostraremos enseguida, si bien esto no invalida el uso de las variables que surgen de las pruebas PISA, sí nos obliga a examinar las posibilidades y limitaciones que estas presentan en cada caso.

En este sentido, un primer conjunto de variables que permite captar ciertos aspectos de la subjetividad estudiantil se refiere a las relaciones entre docentes y alumnos/as (student/teacher relations). En las pruebas se les pregunta a los estudiantes si consideran que 'se llevan bien con sus docentes'; 'los docentes se interesan en ellos'; 'los docentes los escuchan'; 'les ofrecen ayuda' y los 'tratan con justicia'. A cada una de estas preguntas los estudiantes pueden responder: 1) totalmente de acuerdo, 2) de acuerdo, 3) en desacuerdo y 4) totalmente en desacuerdo. Los puntajes más bajos representan buenos vínculos con los docentes y los más altos, relaciones más conflictivas. Como estas variables presentaban un buen nivel de asociación entre sí (Alfa de Cronbach= 0,810) las integramos en un solo índice común denominado relaciones docente/alumno.

Obviamente, estas variables no captan la manera en que los estudiantes se relacionan con sus docentes en toda su complejidad. Es posible que, subyacentes a estas manifestaciones explícitas, existan otras percepciones que constituyan esa relación y que podrían mostrar aspectos distintos. Notoriamente, el cuestionario no incluye preguntas sobre los aspectos estrictamente pedagógicos de la relación: si los docentes explican con claridad, se detienen frente a contenidos no comprendidos, etc. Aun así, las percepciones que captan estas variables tampoco resultan superfluas. Si bien no puede asumirse que representen todos los componentes del vínculo entre docentes y estudiantes, que estos se sientan escuchados y ayudados por los docentes o tratados con justicia por ellos no dejan de constituir aspectos relevantes.

Además de datos sobre la relación entre estudiantes y docentes, las pruebas PISA aportan un conjunto de variables referidas a los sentimientos de pertenencia (sense of belonging) respecto de la escuela. En este caso, se consulta a los y las estudiantes si se sienten 'como extraños' (outsiders) en la escuela; si 'hacían amigos fácilmente'; si sienten que 'pertenecen a la escuela'; 'si se sienten incómodos (awkward) en la escuela'; 'apreciados por otros estudiantes'; 'solos en la escuela', si sienten que la 'escuela es ideal' o si están 'satisfechos con ella'. Este conjunto de variables no mostró un buen nivel de asociación entre sí, de manera que no fue convertida en un solo índice común.

Sin embargo, sí mostraron un buen nivel de asociación aquellas que representaban actitudes negativas o positivas hacia la escuela. Así, compusimos dos indicadores que agrupaban distintas variables. El indicador sentimientos negativos hacia la escuela agrupa las variables 'me siento extraño', 'me siento raro' y 'me siento solo' en la escuela (Alfa de Cronbach=0,798). A su vez, el indicador sentimientos positivos hacia la escuela reúne al resto de las variables que forman parte de la categoría sentimientos de pertenencia que expusimos previamente (Alfa de Cronbach=0,796). Todas estas variables podían ser respondidas según una escala idéntica a la anterior. En el caso de los sentimientos negativos, los puntajes bajos representan mayor aversión a la escuela. En el caso del indicador de sentimientos positivos, estos representan mayor afinidad hacia ella.

3. Nos referimos a la distinción entre la conciencia discursiva, que es donde se elaboran las percepciones de manera reflexiva y que pueden ser verbalizadas, y la conciencia práctica, conformada por disposiciones pre-reflexivas que los actores no controlan plenamente, aunque cuando son requeridas, pueden ser elaboradas reflexivamente por la conciencia discursiva, si bien solo de manera parcial (ver Giddens, 1984 para una discusión más detallada). 
En la misma línea que el caso anterior, estas variables no permiten examinar cabalmente la relación entre las formas de pertenencia que propone la escuela y las que caracterizan a los estudiantes. Por ejemplo, no incluyen preguntas específicas respecto de las normas de disciplina que suelen ser fuente de conflicto. No obstante, aunque no capten todas las dimensiones que podrían caracterizarlas, permiten la estimación de algunas que son ciertamente relevantes (como los sentimientos de pertenencia y satisfacción con la escuela, o las sensaciones de soledad o extrañeza que esta pudiera generar, etc.).

El cuestionario presenta otro conjunto de variables en la categoría actitudes hacia la escuela. Esta incluye afirmaciones como la escuela: 'prepara poco para la vida'; 'es una pérdida de tiempo'; 'es útil para trabajar'; 'sirve para conseguir trabajo'; 'prepara para la universidad' 4 . Los estudiantes podían estar o no de acuerdo con estas afirmaciones en una escala igual a las anteriores. En este caso, los puntajes más bajos podían representar buenos o malos sentimientos respecto de la escuela según el tenor de la afirmación. Estas variables no se encontraban significativamente asociadas entre sí, de manera que no las integramos en un indicador común y serán analizadas independientemente. Aquí, se repiten las posibilidades y limitaciones de los dos casos anteriores. Es posible que no toda la evaluación del conocimiento escolar pase por su utilidad para insertarse en el mercado laboral o continuar los estudios. De hecho, el cuestionario no indaga por el interés, más allá de la utilidad, que pueda generar el saber transmitido por la escuela. Sin embargo, aunque estas variables no permitan evaluar en toda su complejidad la percepción estudiantil del conocimiento que difunde la escuela, al menos permiten estimar algunos de sus aspectos relevantes.

Finalmente, la base de datos presenta un indicador de nivel económico, social y cultural (NESC), que utilizaremos para estimar el estrato social de origen de los estudiantes. Este indicador toma en cuenta el máximo nivel educativo alcanzado por los progenitores, su tipo de inserción laboral y la disponibilidad de bienes durables, tecnológicos y culturales en el hogar (ver OECD, 2013, para más detalles). El indicador se presenta como una variable cuantitativa continua, que en este caso dividimos en terciles para establecer las percepciones de la escuela que tenían, en promedio, los estudiantes de distintos estratos sociales. Al permitir discriminar las percepciones estudiantiles por sector social, esta variable indica si, efectivamente, existen mayores niveles de conflictividad en los sectores con mayor exclusión social. Esto no permite evaluar las causas subyacentes a esas percepciones, por ejemplo, si se vinculan con las frustraciones relacionadas con la imposibilidad de alcanzar el conocimiento que esta imparte o el conflicto con las formas de pertenencia o comportamiento que propone. Aun así, permite estimar, en términos generales, si distintos sectores sociales mantienen mayores o menores niveles de conflicto con la escuela.

\section{Resultados}

En esta sección presentamos el comportamiento de las variables que miden los vínculos entre estudiantes y docentes, los sentimientos de pertenencia a la escuela y la valoración del saber escolar, partiendo de una estimación de su incidencia en general. Luego mostraremos su distribución por país y posteriormente, por sector social. Siguiendo este plan, en el gráfico 1 presentamos la distribución porcentual de los índices relaciones docente/ alumno, sentimientos negativos hacia la escuela, sentimientos positivos hacia la escuela, y las distintas preguntas referidas a las actitudes hacia la escuela.

4. En la categoría se incluían otras variables, pero que no fueron incluidas porque se referían a actitudes muy variadas: 'disfruto de las buenas calificaciones', 'esforzarme es importante', 'me da confianza', etc. 


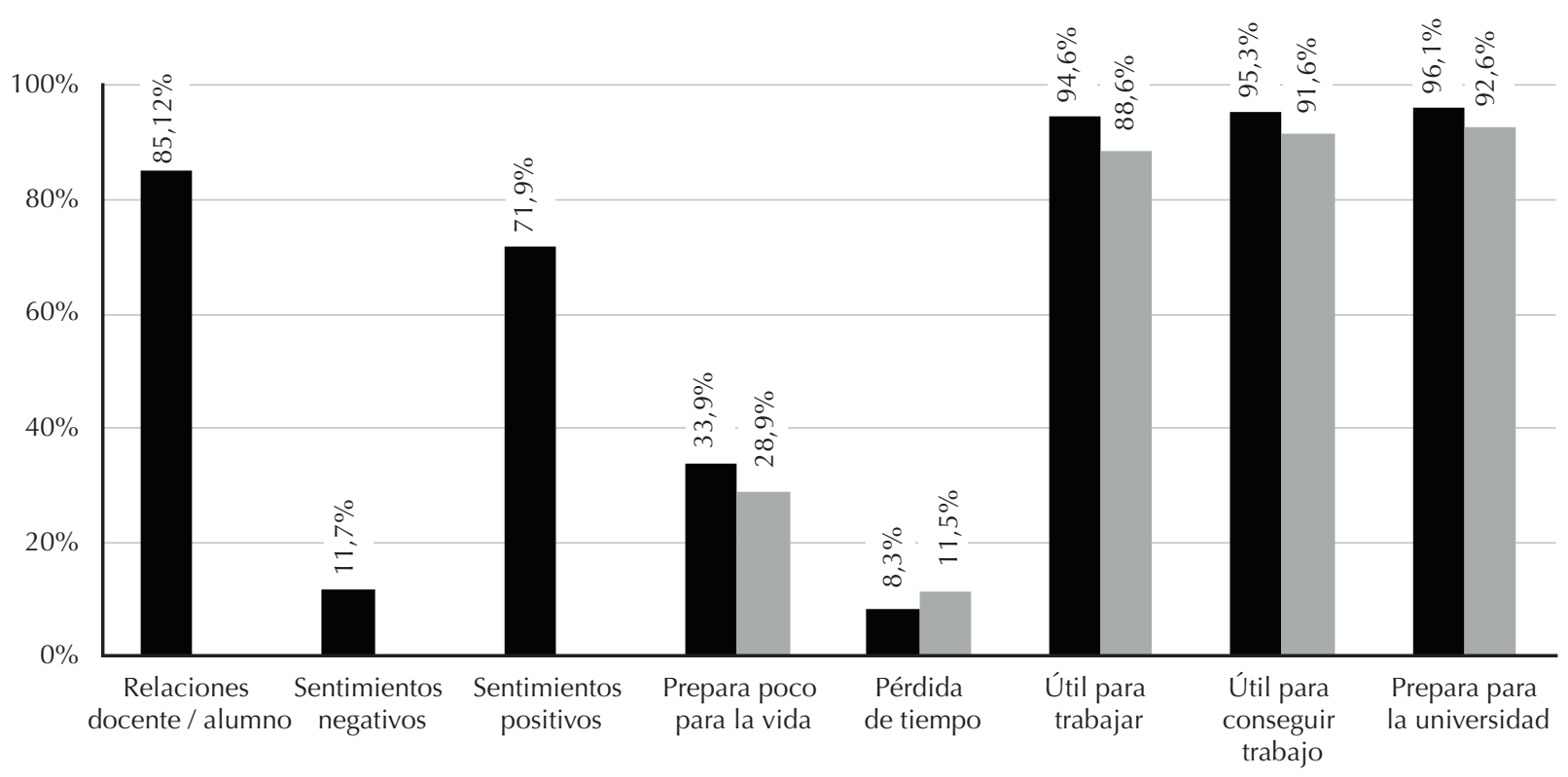

América Latina

OCDE

Gráfico 1. Percepciones de la escuela secundaria (porcentaje de estudiantes de acuerdo o muy de acuerdo). Fuente: PISA 2012.

Los datos revelan que, en estos aspectos, la mayoría de los estudiantes latinoamericanos mantiene percepciones favorables de la escuela. Más del 80\% de los encuestados manifiesta tener buenos vínculos con sus docentes, $70 \%$ expresa sentimientos positivos hacia la escuela y más de $90 \%$ señala que la escuela es útil para el trabajo o los estudios futuros. La variable que expresa un mayor distanciamiento de los estudiantes respecto de la escuela es la que refiere a la preparación general para la vida. En torno a 30\% de los y las estudiantes percibe que la escuela provee una preparación pobre o escasa en este sentido. Es decir que, si bien la mayoría valora el conocimiento que difunde la escuela como insumo para el trabajo y los estudios superiores, un porcentaje minoritario pero significativo no lo considera útil como recurso para un proyecto de vida más integral.

Es claro que cuando tenemos en cuenta que estas variables captan solo algunas dimensiones de las percepciones estudiantiles, no puede asumirse que expresen la totalidad de su experiencia escolar. Como señalamos, debido a las limitaciones del propio instrumento de investigación (la encuesta), el resultado posiblemente enfatice sus dimensiones más explicitas o conscientes y tienda a hacerlas más homogéneas de lo que realmente son. Sin embargo, lo que sugieren estos datos es que, si existe un distanciamiento o extrańamiento entre los estudiantes y la escuela, este no se produce en todas las dimensiones de ese vínculo. Aunque la encuesta no pueda captar estas relaciones en su totalidad, sí indica que, en algunas dimensiones de la experiencia estudiantil, subsisten formas de pertenencia y valoración entre los estudiantes y la escuela.

El gráfico 2 muestra que este resultado tiende a ser común a todos los países de América Latina. Aunque existen algunas diferencias en las que nos detendremos enseguida, en todos ellos la mayoría de los estudiantes tienen percepciones parecidas de sus relaciones con los docentes, manifiestan similares sentimientos respecto de la escuela y valoran de manera equivalente el conocimiento que esta imparte. Así, las tendencias que captábamos para la región se verifican también, aunque con leves fluctuaciones, para cada país. 


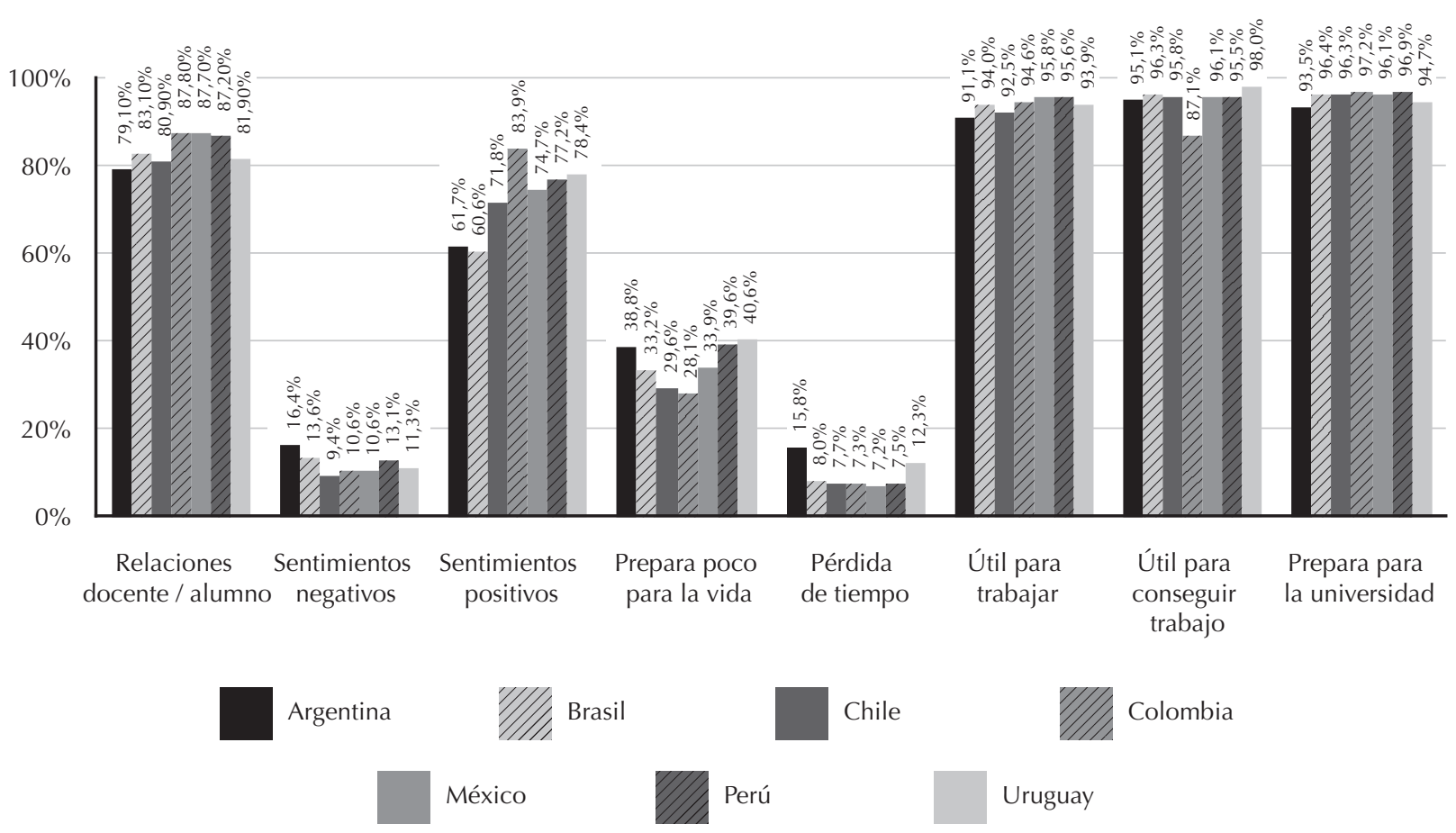

Gráfico 2. Percepciones estudiantiles por pais (porcentaje de estudiantes de acuerdo o muy de acuerdo). Fuente: PISA 2012.

Entre los países que presentan algunas diferencias al promedio se destaca Argentina y, en menor medida, Brasil, Perú y Uruguay. El primero posee una menor proporción de estudiantes que manifiestan tener buenas relaciones con sus docentes (aunque estos siguen siendo la gran mayoría). Asimismo, es el país en donde las minorías de estudiantes que tienen sentimientos negativos hacia la escuela piensan que esta prepara poco para la vida y que es una pérdida de tiempo son más numerosas. También es el país donde menos estudiantes creen que la escuela es útil para trabajar, ayuda a conseguir trabajo o preparar para la universidad (aunque los que creen que sí lo es siguen siendo una notable mayoría).

Brasil, Perú y Uruguay, por su parte, muestra un patrón menos consistente. En los tres países, las minorías que tienen sentimientos negativos hacia la escuela, o que piensan que esta no prepara para la vida o que es una pérdida de tiempo son mayores que el promedio de la región. Sin embargo, a diferencia de Argentina, no son países donde la proporción de estudiantes que cree que la escuela es un medio adecuado para continuar los estudios o ingresar al mundo laboral es consistentemente menor que el promedio regional.

Es decir que, si bien estos tres casos presentan algunas diferencias, no muestran patrones muy distintos al resto de los países. En rigor, más allá de que en el caso de Argentina las diferencias son más consistentes, tampoco tienen una magnitud que las distinga sustantivamente del resto de Latinoamérica ${ }^{5}$. Así, lo que surge de estos cálculos es una notable homogeneidad regional que, más allá de los matices, sugiere que en América Latina existen aspectos de la experiencia escolar que son comunes a la mayoría de los estudiantes.

5. La evidencia disponible no permite conjeturas claras sobre las razones que podrían explicar estas variaciones. En el caso de Argentina, su sistema educativo ha sufrido un deterioro, perdiendo su capacidad de actuar como un mecanismo de igualación social (Guadagni, Cuervo, y Sica, 2002), lo que podría explicar las percepciones más negativas que en el resto de la región. Sin embargo, esta misma tendencia no se verifica en los otros países, donde incluso en algunos casos, como Brasil, el sistema educativo ha mejorado su capacidad inclusiva. Explicar estos patrones requeriría una investigación adicional que escapa a las posibilidades de este trabajo. 
Ahora bien, cuando atendemos a las diferencias por sector social, este panorama general no sufre grandes modificaciones, pero sí se ponen en evidencia matices interesantes. El gráfico 3 indica que, en todos los estratos sociales, son mayoría los estudiantes que manifiestan tener buenos vínculos con sus docentes. Solo una minoría mantiene sentimientos negativos hacia la escuela, mientras la mayoría expresa sentimientos positivos. Asimismo, una minoría, aunque significativa, indica que la escuela prepara poco para la vida en general, y una minoría muy pequeña cree que asistir a la escuela es una pérdida de tiempo. A su vez, casi todos los estudiantes creen que la escuela prepara para el trabajo y es útil para obtenerlo, y también para continuar los estudios superiores. De manera que, en principio, la comparación de las variables por estrato indica que en todos ellos se mantienen las mismas tendencias que encontramos a nivel general.

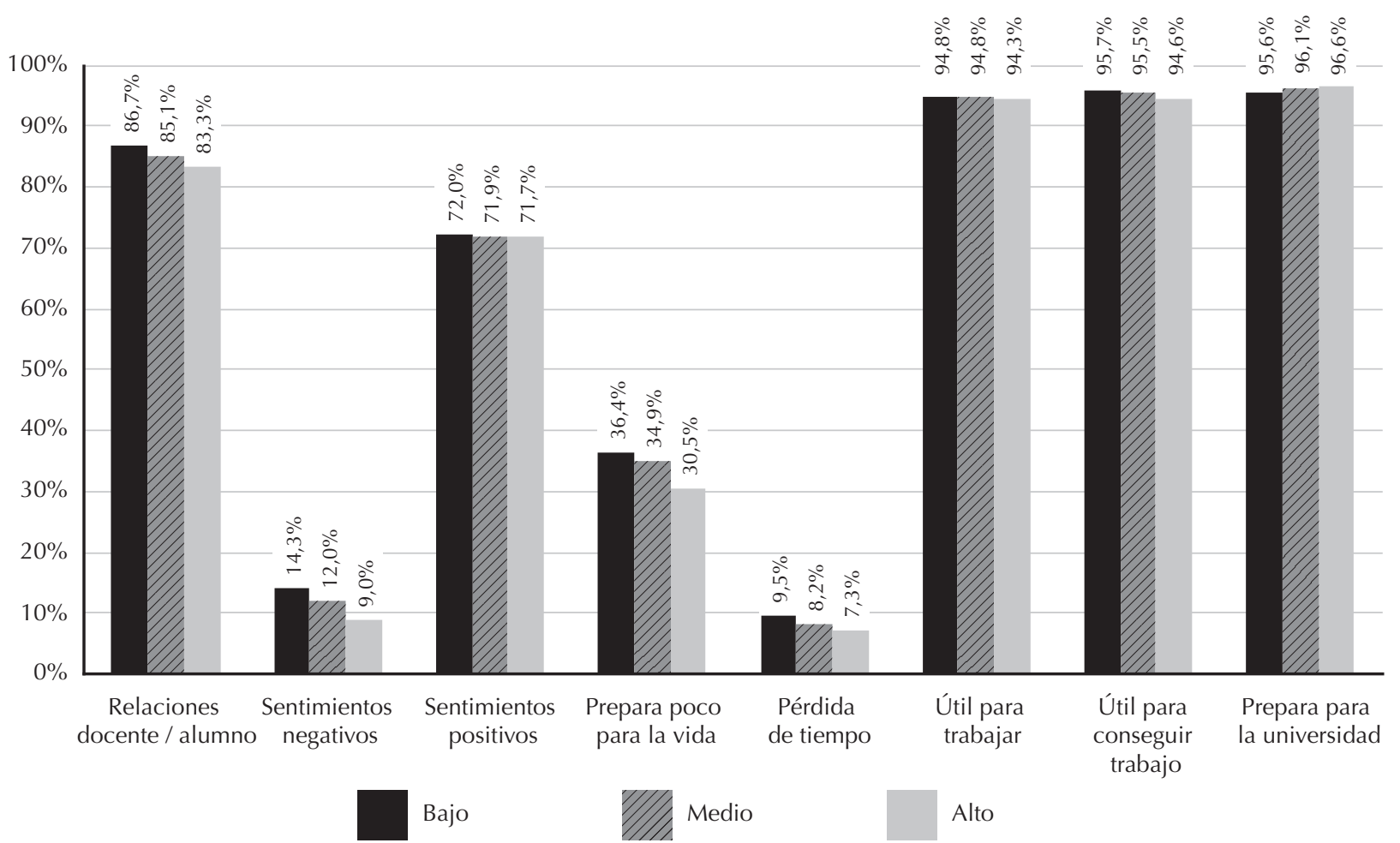

Gráfico 3. Percepciones estudiantiles por estrato social (porcentaje de estudiantes de acuerdo o muy de acuerdo). Fuente: PISA 2012.

Pero si estas tendencias generales confirman lo que sabíamos a partir de los gráficos precedentes, las diferencias relativas que surgen al comparar la incidencia de cada variable por sector social revelan matices interesantes. Algunas de las variables muestran que, entre los estratos sociales con mayores carencias materiales y culturales, la desvalorización y extrañamiento respecto de la escuela tiende a ser mayor. No obstante, otras variables revelan percepciones similares o incluso levemente más favorables que en otros sectores sociales, sugiriendo que esta propensión no es homogénea para todas las dimensiones de la experiencia escolar.

En específico, los datos indican que existen más estudiantes de los estratos más pobres que creen que la escuela sirve poco para la vida, experimentan sentimientos negativos hacia ella y consideran que asistir es una pérdida de tiempo. A su vez, es menor la proporción de alumnos de este estrato social que cree que la escuela prepara para la universidad. Sin embargo, es algo mayor la proporción de estudiantes de ese grupo que cree que 
la escuela secundaria prepara para el trabajo. Además, la mayoría que experimenta sentimientos positivos hacia la escuela es levemente mayor en este sector y, notablemente, es también relativamente más amplia la mayoría que manifiesta tener buenos vínculos con los docentes.

De esta manera, los datos sugieren que ciertas dimensiones de la experiencia escolar son efectivamente más negativas para los estratos sociales más bajos que para los más altos, como lo suponían las premisas referidas a los sectores con mayores dificultades para integrarse socialmente. No obstante, los datos también indican que esa experiencia es heterogénea, y que como preparación para el trabajo y en la vinculación con los docentes, resulta satisfactoria para una proporción similar o incluso levemente mayor de estudiantes de esos sectores que de otros.

Es probable que esta heterogeneidad de la experiencia efectivamente se vincule a la posición en la estructura social. Por ejemplo, que la mayor proporción de estudiantes de los estratos más bajos que manifiesta tener sentimientos positivos hacia a la escuela o buenos vínculos con los docentes resulta de que estos se presentan como un espacio de contención que no encuentran en otros ámbitos (Reimers, 2000). O que la menor proporción de estudiantes de este estrato que considera que la escuela prepara para la universidad se vincule al hecho de que consideran lejana la posibilidad de seguir estudios superiores (y perciben a la inserción laboral como más realista y por eso la mayoría que señala a la escuela como preparación para el trabajo es algo mayor que en otros estratos).

Pero si, en definitiva, estos matices marcan que efectivamente existen variaciones en las percepciones de los estudiantes según su origen social, al menos en los aspectos que son captados por estas variables, esto no supone una percepción homogéneamente más negativa en los sectores más desfavorecidos. Lo que sí muestra es que la valoración de los varios aspectos de esa experiencia es diversa entre estudiantes de distinto origen social.

En síntesis, si bien las variables que examinamos no permiten resultados conclusivos, sí sugieren que ciertos aspectos de la experiencia escolar generan percepciones favorables entre los estudiantes. Algunas dimensiones de su relación con los docentes, ciertas formas de pertenencia escolar y determinados saberes impartidos por la escuela aún resultan relevantes para ellos.

Por las razones que ya adelantamos, estos resultados no habilitan conclusiones definitivas. Algunas de las investigaciones precedentes basadas en estudios de caso sugieren que subyacentes a estas percepciones tal vez existan otras que no son plenamente coincidentes con las que surgen de las pruebas PISA. Así, lo que parece quedar pendiente para lograr una mayor comprensión de la vivencia estudiantil de la escuela es un análisis más exhaustivo y representativo de cuáles aspectos de la experiencia escolar siguen generando identificación y cuáles, extrańamiento en la etapa avanzada de la modernidad.

\section{Discusión y conclusiones}

El principal propósito de este artículo consistió en indagar sobre las percepciones que tenían los estudiantes latinoamericanos de sus escuelas secundarias como producto de los cambios introducidos por la modernidad avanzada. A partir del análisis de estas percepciones, pretendimos evaluar algunas de las premisas que han guiado múltiples investigaciones en el campo. De manera resumida, puede identificarse una premisa general (que la modernidad avanzada generaría vínculos crecientemente conflictivos entre los estudiantes y la escuela), apoyada en cuatro premisas complementarias: i) que los cambios en los modelos de autoridad escolar generados por el carácter reflexivo que toman las relaciones interpersonales en la modernidad avanzada habrían resultado en vínculos más conflictivos entre docentes y estudiantes; ii) que las nuevas formas de comunicación y sociabilidad juvenil resultarían en un extrańamiento entre los estudiantes y la escuela; iii) que la celeridad con la que se acumula y circula la información y el conocimiento en la modernidad avanzada haría obsoletos los saberes y modalidades 
de aprendizaje promovidos por la escuela, y iv) que los avances cognoscitivos y tecnológicos introducidos por la modernidad avanzada incrementarían los grados de exclusión de los sectores más vulnerables, aumentando los niveles de conflicto entre estos y la escuela.

Como hemos señalado, los datos disponibles no permiten evaluar exhaustivamente las sofisticadas elaboraciones teóricas de las que surgen estas premisas. Sin embargo, aun con limitaciones, permiten ponderar algunas dimensiones importantes y sugerir maneras de refinar estos puntos de partida.

Respecto de la primera premisa, el indicador sobre los vínculos entre docentes y estudiantes permite estimar las percepciones de los alumnos sobre el interés que manifiestan los docentes por ellos, el grado en que se sienten asistidos y escuchados por sus profesores, y el nivel de justicia con que se sienten tratados por estos.

Si bien los datos de las pruebas PISA muestran que los estudiantes tienen percepciones predominantemente favorables en este sentido, este dato por sí solo no permite conclusiones definitivas. De hecho, algunos de los estudios de caso que citamos muestran que, al tener en cuenta otras dimensiones de la experiencia escolar, como los niveles de exigencia académica, la percepción de los vínculos con los docentes puede ser distinta. Pueden sentirlos como fríos y distantes. Es decir que, si bien en algunas dimensiones la relación docente/estudiante podría generar percepciones favorables, en otras tal vez no sea percibida en los mismos términos.

La indagación de la segunda premisa muestra una tendencia similar. Las variables contenidas en las pruebas PISA estiman las sensaciones de pertenencia que los estudiantes mantienen respecto de la escuela. Específicamente, establecen si los estudiantes la perciben como 'ideal', están 'satisfechos' y sienten que 'pertenecen' a ella. O si se sienten solos o extrańos en la escuela. Los resultados muestran que en estos aspectos predominan las percepciones positivas. No obstante, al contrastar con los resultados de los estudios de caso, este resultado sugiere, nuevamente, la multidimensionalidad de la experiencia escolar. Por ejemplo, en algunos estudios se revela que, cuando se indaga sobre los sistemas disciplinarios o el interés que suscita la escuela, esta puede ser percibida como excesivamente rígida o aburrida.

El análisis de la tercera premisa arroja un resultado similar. Las variables contenidas en las pruebas PISA estiman las percepciones de los estudiantes respecto del conocimiento transmitido por la escuela. En específico, consideran la utilidad de ese conocimiento para el trabajo, continuar los estudios superiores o desarrollar un proyecto de vida. En general, los estudiantes consideran el conocimiento transmitido por la escuela útil, aunque en el último caso (la vida en general) la proporción de estudiantes que lo hace es menor. Sin embargo, no puede considerarse que la utilidad que brinda el conocimiento sea el único parámetro que interviene en las percepciones estudiantiles. De hecho, uno de los estudios de caso revela que aun cuando se considere relevante, la percepción de que ese saber no es distribuido homogéneamente en las distintas escuelas puede llevar a una evaluación negativa. Es decir, no solo se evalúa la utilidad del conocimiento, sino la equidad en su distribución.

El análisis de la última premisa también muestra resultados matizados. Si bien la percepción de la escuela es bastante homogénea entre distintos sectores sociales, algunos parámetros muestran que ciertos aspectos son más conflictivos para los sectores de menores recursos. Por ejemplo, en el estrato más bajo, hay minorías más numerosas que mantienen sentimientos negativos hacia la escuela o la perciben como una pérdida de tiempo. Sin embargo, esto convive con mayorías levemente más numerosas que perciben favorablemente sus vínculos con los docentes o que valoran su experiencia escolar como positiva. Esto sugiere una heterogeneidad de la experiencia escolar en estos sectores. En coincidencia con lo que plantea la cuarta premisa, en algunos aspectos la experiencia escolar de estos sectores es más conflictiva que en otros. Pero en tensión con ella, en otras dimensiones resulta más favorable. Este resultado no parece conducir entonces a una confirmación o refutación de esta premisa. En cambio, sugiere la necesidad de un análisis más cuidadoso respecto de cuáles aspectos de la experiencia generan lejanía y cuáles, pertenencia. 
Para terminar, es justo decir que varias de las teorías de las que surgen las premisas que hemos evaluado en este trabajo plantean justamente este carácter multidimensional de la experiencia escolar. Sin embargo, tienden a centrarse en la variación de esa experiencia entre distintos sectores sociales, y también a enfatizar sus aspectos más conflictivos. En comparación con esa perspectiva, los datos de las pruebas PISA muestran que la escuela también produce experiencias que son valoradas y generan sentimientos de pertenencia. Es decir que, al menos en algunas dimensiones, proporciones significativas de estudiantes latinoamericanos mantienen percepciones favorables de la escuela. Aunque con matices, esta es una tendencia general que atraviesa varios países y sectores sociales en la región.

Sin embargo, las limitaciones de estos datos no permiten asumir a estas percepciones como únicas. Al tomar en cuenta lo que revelan varios estudios de caso, se plantea la alternativa de que subyacentes a estas percepciones favorables subsistan otras en conflicto con ellas. De esta manera, surge la posibilidad de que en un mismo grupo de estudiantes coexistan experiencias positivas y negativas de la escuela, o incluso que esa misma variación forme parte de la experiencia de un mismo estudiante.

En definitiva, lo que este balance sugiere es que nuestra comprensión de la experiencia escolar en la modernidad avanzada podría enriquecerse introduciendo premisas que permitan identificar esta complejidad. Es decir, incorporar preguntas o parámetros que permitan discernir cuáles aspectos de la experiencia escolar se han vuelto conflictivos y cuáles siguen contribuyendo a la formación de conocimientos, formas de pertenencia y relaciones socialmente valoradas. Si bien los estudios de caso aportan a ese proyecto por su mayor sensibilidad a esos matices, tienen la limitación de no revelar cuán extendidas podrían ser esas variaciones. En este sentido, son también relevantes las aproximaciones cuantitativas que habiliten un reconocimiento más extendido de las dimensiones positivas y negativas de la experiencia estudiantil. Aunque aquí no podemos plantear una agenda de investigación completa, lo que al menos sugiere este recorrido es que posiblemente sea esta combinación metodológica la que nos permita avanzar más en la comprensión de esa complejidad.

El artículo original fue recibido el 10 de diciembre de 2019

El artículo revisado fue recibido el 14 de julio de 2020

El artículo fue aceptado el 29 de septiembre de 2020

\section{Bibliografía}

Abramovay, M. (2003) Violencia na escola. America Latina e Caribe. Brasilia, Brazil: UNESCO.

Bauman, Z. (2005). Education in liquid modernity. Review of Education, Pedagogy \& Cultural Studies, 27(4), 303-317. https://doi.org/10.1080/10714410500338873

Burbules, N., y Torres, C. (2013). Globalization and education: An introduction. En N. C. Burbules \& C. A. Torres (Eds.), Globalization and Education: Critical Perspectives (pp. 1-26). Nueva York, NY: Taylor and Francis.

Calicchio, T., de León, E, Gutiérrez, M., Lorenzo, M., \& Radakovich, R. (2004). Cultura juvenil y educación media superior en el Uruguay. Aportes para la reflexión y transformación de la educación media superior, (26).

Castel, R., (1997) Las metamorfosis de la cuestión social. Buenos Aires, Argentina: Paidós.

Coleman, J. (1961). The Adolescent Society. The social life of the teenager and its impact on education. Glencoe, Escocia: Free Press.

Cicourel, A. (1964). Method and measurement in sociology. Londres, Reino Unido: The Free Press of Glencoe.

de Giraldo, L. y Mera, R. (2000). Clima social escolar: Percepción del Estudiante. Colombia Médica, 31(1), 23-27.

Recuperado de http://uvsalud.univalle.edu.co/colombiamedica/index.php/comedica/article/view/148 
Dubet, F. (1995) Sociologie de l'expérience. París, Francia: Seuil.

Dubet, F. y Martuccelli, D. (1997). En la escuela. Sociología de la experiencia escolar. Buenos Aires, Argentina: Losada.

Dubet, F. (2002). Le déclin de l'institution. París, Francia: Seuil.

Dubet, F. (2009) Las paradojas de la integración escolar. Espacios en Blanco. Revista de Educación, 19, 197-214. Recuperado de http:/www.espaciosenblanco.unicen.edu.ar/pdf/numerorosanterior/Revista_Espacios_en_Blanco_N19.pdf

Dubet, F. (2011). 'Mutacôes cruzadas: a cidadania e a escola'. Revista Brasileira de Educacâo. 16(47), 289-305. https://doi.org/10.1590/S1413-24782011000200002

Duschatzky, S. y Corea, C. (2002). Chicos en banda. Los caminos de la subjetividad en el declive de las instituciones. Buenos Aires, Argentina: Paidós.

Dussel, I., Brito, A., Nuñez, P., \& Litichever, L. (2006). La escuela media argentina: estudio nacional sobre las opiniones de los jóvenes y docentes. Buenos Aires, Argentina: Santillana.

Fundación SES. (2018). Relevamiento nacional sobre percepción de las y los adolescentes y jóvenes respecto a la escuela media. Buenos Aires, Argentina: Fundación SES.

García Canclini, N. (1995). Consumidores y ciudadanos. Conflictos multiculturales de la globalización. Ciudad de México, México: Grijalbo.

Giacobbe, C. y Merino, L. (2015). Los alumnos y la autoridad docente ¿Crisis de sentido? Educación, Formación e Investigación, 1(1), 1-22. Recuperado de http://ppct.caicyt.gov.ar/index.php/efi/article/view/6303/5678

Giddens, A. (1984). La Constitución de la sociedad. Buenos Aires, Argentina: Amorrortu.

Giddens, A. (1997). Las consecuencias de la modernidad. Buenos Aires, Argentina: Amorrortu.

Guadagni, A., Cuervo, M., \& Sica, D. (2002). En busca de la escuela perdida. Educación, crecimiento y exclusión social en la Argentina del siglo XX. Buenos Aires, Argentina: Siglo XXI.

Krüger, N. (2019). La segregación por nivel socioeconómico como dimensión de la exclusión educativa. 15 años de evolución en América Latina. Archivos Analiticos de Politicas Educativas, 27(8), 1-37. https://doi.org/10.14507/epaa.27.3577

Lewkowicz, I. (2004). Pensar sin estado. La subjetividad en la era de la fluidez. Buenos Aires, Argentina: Paidós.

Llinás, P. (2009a). Imágenes y sentidos de la experiencia escolar: percepciones de los estudiantes sobre la escuela secundaria. Propuesta Educativa, (32), 95-104. Recuperado de http://propuestaeducativa.flacso.org.ar/wp-content/ uploads/2019/12/articulo_llinas.pdf

Llinás, P. (2009b). Sentidos de la experiencia escolar en la escuela secundaria: percepciones de los alumnos en cuatro jurisdicciones argentinas. Páginas de Educación, 2(1), 91-114. https://doi.org/10.22235/pe.v2i1.704

Manzano, Valeria. (2010). Ha llegado la “nueva ola”: música, consumo y juventud. En I. Cosse, K. Felitti, y V. Manzano (Eds.), Los '60 de otra manera. Vida cotidiana, género y sexualidades en la Argentina. Buenos Aires, Argentina: Prometeo.

Martuccelli, D. (2009). La autoridad en las salas de clase. Problemas estructurales y márgenes de acción. Diversia, 1, 99-128. Recuperado de https://www.cidpa.cl/?p=308

Martuccelli, D. (2016). Condición adolescente y ciudadanía escolar. Educação \& Realidade, 41(1), 155-174. https://doi.org/10.1590/2175-623660050

Martuccelli, D. (2017). La nueva dinámica de la condición social moderna. Revista de Sociología, (32), 89-105. https://doi.org/10.5354/0719-529x.2017.47887

Metiffogo, D. y Sepúlveda, R. (2005). Trayectorias de vida de jóvenes infractores de la ley. Santiago, Chile: Universidad de Chile. Recuperado de http://repositorio.uchile.cl/bitstream/handle/2250/151816/4-Trayectorias-de-vida. pdf?sequence $=18$ isAllowed $=y$

Merle, P. (2012). La ségrégation scolaire. París, Francia: La Découverte.

Meza, A. (2017). Percepciones de los/las estudiantes sobre la organización de una escuela normal. Memorias del Congreso Nacional de Investigación Sobre Educación Normal, 1(1), 1-11. Recuperado de http://www.conisen.mx/memorias/memorias/3/C200117-J082.docx.pdf

Miranda López, F. (2012). Los jóvenes contra la escuela. Un desafío para pensar las voces y tiempos para América Latina. Revista Latinoamericana de Educación Comparada, 3(3), 71-84. Recuperado de http://www.saece.com.ar/relec/revistas/3/art6.pdf 
OECD. (2013). PISA 2012 Results: Excellence Through Equity: Giving Every Student the Chance to Succeed (Volume II). París, Francia: OECD Publishing. https://doi.org/10.1787/9789264201132-en

OECD. (2016). PISA 2015 Results (Volume I): Excellence and Equity in Education. París, Francia: OECD Publishing. https://doi.org/10.1787/9789264266490-en

Perelman, L. (1992). Schools out: Hyperlearning, the new technology, and the end of education. Nueva York, NY: Avon Books.

Richards, C. (2009). Por qué me fui de la escuela. Testimonios de jóvenes que viven la interrupción de su trayectoria educativa. En C. Eroles y C. Hirmas (Comps.), Experiencias educativas de segunda oportunidad. Lecciones desde la práctica innovadora en América Latina. Santiago, Chile: ORELAC.

Reimers, F. (2000). Educación, desigualdad y opciones de política en América Latina en el siglo XXI. Revista Latinoamericana de Educación, 23, 21-50. https://doi.org/10.35362/rie2301006

Rodríguez, E. (2002). Cultura juvenil y cultura escolar en la enseñanza media del Uruguay de hoy: un vínculo a construir. Ultima Década, 10(16), 53-94. https://doi.org/10.4067/S0718-22362002000100003

Saintout, F. (2007). Jóvenes e incertidumbres. Percepciones de un tiempo de cambios: familia, escuela, trabajo y política (Tesis de doctorado inédita). Facultad Latinoamericana de Ciencias Sociales, Buenos Aires.

Sánchez, M. (2006). Disciplina, autoridad y malestar en la escuela. Revista Iberoamericana de Educación. 41(1). Recuperado de https://rieoei.org/historico/jano/opinion38.htm

Sapiains, R. y Zuleta, P. (2001). Representaciones sociales de la escuela en jóvenes urbano-populares desescolarizados. Ex-cuela y juventud popular: la escuela desde la desescolarización. Ultima Década, 9(15), 53-72. Recuperado de https://ultimadecada.uchile.cl/index.php/UD/article/view/56515/59835

Sennet, R. (1998). The corrosion of character. Nueva York, NY: Norton.

Susmel, N., (2012). Argentina: Pobreza, desigualdad de oportunidades y políticas públicas. En Pobreza, desigualdad de oportunidades y politicas públicas en América Latina. Río de Janeiro, Brasil: Konrad Adenauer Stiftung. Recuperado de https://www.kas.de/c/document_library/get_file?uuid=d82f6024-1a73-0a65-6ea4530e5bd750d3\&groupId $=252038$

Tapia, G., Pantoja, J., y Fierro, M. C. (2010) ¿La escuela hace la diferencia? El abandono de la escuela secundaria en Guanajuato, México. Revista Mexicana de Investigación Educativa, 15(44), 197-225. Recuperado de https://www.comie.org.mx/revista/v2018/rmie/index.php/nrmie/article/view/428

Tenti, E. (2000, 7-9 de junio). Culturas juveniles y cultura escolar. Documento presentado en el seminario Escola Jovem: un novo olhar sobre o ensino médio. Ministerio de Educación, Brasil.

Tenti, E. (2014). Viejas y nuevas formas de autoridad docente. Revista Todavía, 7, 1-4. Recuperado de http://blogs.unlp.edu.ar/pec/files/2014/11/Tenti-Fanfani-Viejas-y-nuevas-formas-de-autoridad-docente.pdf

Tiramonti, G. y Minteguiaga, A., (2007). 'Una nueva cartografía de sentidos para la escuela.' En: Tiramonti, G. (comp.), La trama de la desigualdad educativa: mutaciones recientes en la escuela media. Buenos Aires, Argentina: Manantial. 